\title{
A crack arrest methodology based on Bazant's parameter to estimate threshold fretting conditions
}

\author{
José Araújo, Raphael Cardoso, Jorge Ferreira, Fábio Castro and Estarle Campos \\ Department of Mechanical Engineering, University of Brasília, 70910-900, DF, Brazil
}

\begin{abstract}
Fracture mechanics-based methodologies, considering either the KitagawaTakahashi (K-T) or El Haddad threshold diagrams, have been used to estimate crack arrest under fretting conditions. It is claimed in this work that the success of these diagrams to correlate experimental data depends on material behaviour. Therefore, a more general methodology based on the Bazant's threshold diagram is proposed here. An appealing feature of such a diagram is that, depending on the value of an adjustable parameter, it may collapse into the K-T or El Haddad diagrams as limiting cases. A preliminary assessment of the model was carried out with available sizes of short arrested cracks observed in cylindrical contacts under partial slip regime. The best estimates were obtained when the upper limit of the Bazant's diagram (K-T diagram) was considered.
\end{abstract}

\section{Introduction and methodology}

Fretting can be defined as a small oscillatory movement between contacting surfaces which occurs in engineering assemblies subjected to vibration or cyclic stress. This micro-slip induces surface damage and favour the premature nucleation and subsequent growth of cracks. Fracture mechanics-based methodologies, considering either the Kitagawa-Takahashi (K-T) or El Haddad threshold diagrams, have been used to estimate crack arrest under fretting conditions [1-3]. It is claimed in this work that the success of these diagrams to correlate experimental data depends on material behaviour. Therefore, a more general methodology based on the Bazant's threshold diagram is presented here.

Figure 1 shows a scheme of the proposed methodology, which is based on (i) the calculation of an effective stress intensity factor range defined as $\Delta K_{\text {eff }}=\sqrt{\Delta K_{\mathrm{I}}^{2}+\Delta K_{\mathrm{II}}^{2}}$, where $\Delta K_{\mathrm{I}}$ and $\Delta K_{\mathrm{II}}$ are respectively the mode I and II stress intensity factor ranges, and on (ii) a further comparison with the Bazant's threshold curve [4] expressed as:

$$
\Delta K_{t h}(b)=\Delta K_{0} \cdot\left[1+\left(b_{0} / b\right)^{\gamma / 2}\right]^{-1 / \gamma}
$$

where $\Delta \mathrm{K}_{0}$ is the threshold stress intensity factor range for long crack propagation, $\mathrm{b}$ is the crack size, $b_{0}$ is a first approximation for the transition between short and long cracks, and $\gamma$ is an adjustable parameter, which can be obtained by fitting experimental data. When $\gamma=2$ and $\gamma=\infty$, Eq. (1) provides the El Haddad and the Kitagawa-Takahashi threshold curves, respectively. Crack arrest is estimated if the condition $\Delta K_{\text {eff }}=\Delta K_{t h}$ occurs along the crack path.

This is an Open Access article distributed under the terms of the Creative Commons Attribution License 4.0, which permits unrestricted use, distribution, and reproduction in any medium, provided the original work is properly cited. 


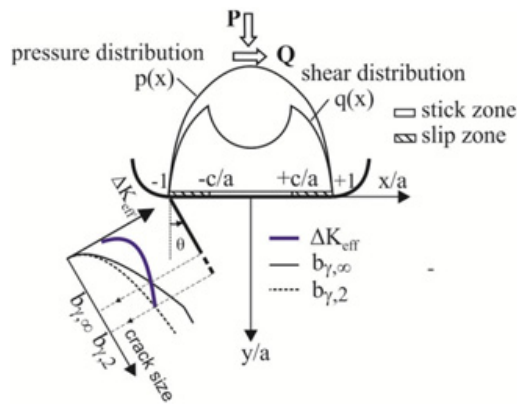

Figure 1. Schematic illustration of the crack arrest methodology.

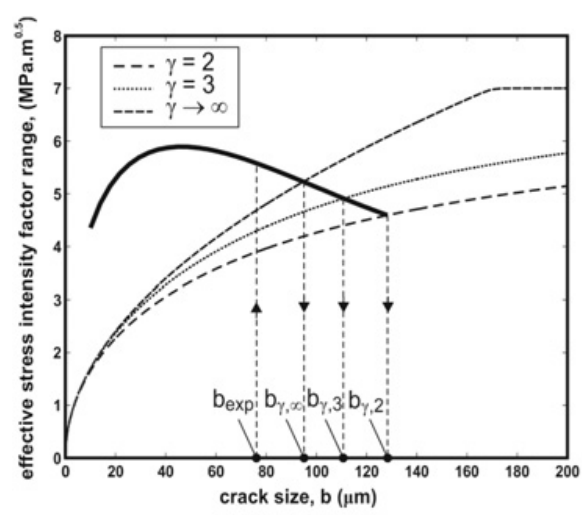

(a)

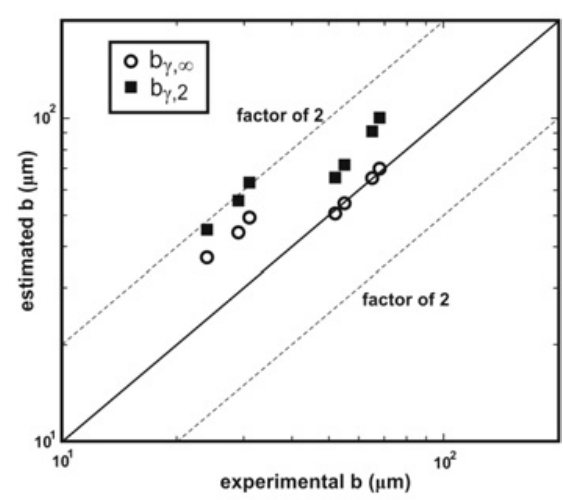

(b)

Figure 2. (a) Illustration of the method $(P=540 \mathrm{~N} / \mathrm{mm}$ and $\mathrm{Q}=278 \mathrm{~N} / \mathrm{mm})$; (b) Estimated vs observed size of short arrested cracks.

\section{Assessment and conclusions}

A preliminary assessment of the model was carried out with available sizes of short arrested cracks observed in cylindrical contacts under partial slip regime [2]. The specimens and cylindrical pads were made of AISI 1034 and 52100 steels, respectively. Figure 2 shows the results considering a crack inclined $30^{\circ}$ with respect to the contact surface. The best estimates were obtained for $\gamma=\infty$.

However, it is known that in previous works [3] involving fretting problems in aluminium alloys better results were obtained using the El Haddad's curve $(\gamma=2)$. Therefore, neither Kitagawa-Takahashi nor El Haddad's curves should be considered as "the best" threshold line. Instead, in order to improve the accuracy of this class of short crack arrest methodologies, a previous knowledge of the Bazant's parameter based on experimental data for the material studied is necessary. Notice that, while the use of the K-T $(\gamma=\infty)$ threshold line provided the best estimates for the data (steel specimens) considered in this work, the use of such curve to estimate short crack arrest for aluminium data [3], could provide unsafe estimates.

\section{References}

[1] J. A. Araújo, D.Nowell, Int. J. Fat., Analysis of pad size effects in fretting fatigue using short crack arrest methodologies 21, 947-956 (1999) 
[2] S. Fouvry, D. Nowell, K. Kubiak, D.A. Hills, Eng Fract Mech, Prediction of fretting crack propagation based on a short crack methodology 75, 1605-1622 (2008)

[3] D.Dini, D. Nowell, I. Dyson, Tribol Int, The use of notch and short crack approaches to fretting fatigue threshold prediction: Theory and experimental validation 39, 1158-1165 (2006)

[4] Z.P. Bazant, Int J Fract, Scalling of quasibrittle fracture: asymptotic analysis 83, 19-40 (1977) 